\title{
Local dissipative Hamiltonian description of reversible reaction networks
}

\author{
Irene Otero-Muras ${ }^{1}$, Gábor Szederkényi ${ }^{2, *}$,
} Antonio A. Alonso ${ }^{1}$, Katalin M. Hangos ${ }^{2}$

${ }^{1}$ Department of Process Engineering, IIM-CSIC, Spanish Council for Scientific Research,

Eduardo Cabello 6, 36208 Vigo, Spain

Tel: +34986231930

Fax: +36986292762

e-mail: ireneotero@iim.csic.es, antonio@iim.csic.es

${ }^{2}$ Process Control Research Group, Systems and Control Laboratory

Computer and Automation Research Institute,

Hungarian Academy of Sciences

H-1518, P.O. Box 63, Budapest, Hungary

Tel: +36 12796000

Fax: +3614667503

e-mail: szeder@sztaki.hu, hangos@scl.sztaki.hu

*corresponding author 


\begin{abstract}
In this letter we show that closed reversible chemical reaction networks with independent elementary reactions admit a global pseudoHamiltonian structure which is at least locally dissipative around any equilibrium point. The structure matrix of the Hamiltonian description reflects the graph topology of the reaction network and it is a smooth function of the concentrations of the chemical species in the positive orthant. The physical interpretation of the description is briefly explained and two illustrative examples are presented for global and local dissipative Hamiltonian description, respectively.
\end{abstract}

Keywords: Hamiltonian systems, Nonlinear systems, Reaction kinetic systems, Reaction networks

\title{
1 Introduction
}

The class of Hamiltonian systems as a special type of passive systems have gained significant interest during the last decade [5]. This interest is mainly originated from the fact that Hamiltonian systems have a number of advantageous properties from a control theoretic point of view. However, the Hamiltonian description of dynamical systems in an appropriate coordinates system is also extremely useful for the deep understanding of the underlying physical processes and the complex relations between the system's structure and the preservation or dissipation of energy-like quantities. Beside pure mechanical systems, well-known examples of Hamiltonian systems are LC-circuits [10, 4], electromechanical models [13] and even certain classes of distributed process systems [9]. The possibility to transform nonlinear control systems to Hamiltonian form via feedback has been studied in a recent paper [2].

A more general system class is the set of pseudo-Hamiltonian systems [3], where the algebraic system structure is the same as in the case of Hamiltonian systems, but the invariance or the decreasing nature of the Hamiltonian function cannot be proved globally from the properties of the structure matrix of the system. In [17], an effective general algorithm is presented for obtaining 
the approximate dissipative Hamiltonian realization and constructing local Lyapunov functions for continuous time nonlinear systems.

One of the most significant achievements in the study of the dynamical properties of complex chemical reaction systems is [7], where (among other important results) the global stability of so-called 'deficiency zero' reaction networks is proved with a given Lyapunov function. It is important to note that the deficiency zero property is a structural feature of a certain class of reaction networks, therefore their stability does not depend on the system parameters. These ideas were recalled, extended and put into a control theoretic framework in [15]. In agreement with [7] (as they fall into the deficiency zero class) the closed and reversible reaction networks with independent elementary reactions are proved to be globally stable [8]. The thermodynamical foundations of the passivity properties of process systems (into which class reaction kinetic systems also belong) were defined in [18] and [1], where the storage function is derived from the entropy.

It is shown in [3] and [11] that Lyapunov-stable nonlinear systems can be algorithmically transformed to a special Hamiltonian form, but in this case, the structure matrix is generally nonsmooth at the equilibrium point, and not much physical insight is gained about the system structure.

Based on the above mentioned results, the aim of this short paper is to show that closed reversible reaction networks admit a pseudo-Hamiltonian structure where the structure matrix is smooth in the positive orthant of the concentration space and it is negative definite in a neighborhood of any equilibrium point if the elementary reactions are independent.

\section{Basic notions}

\subsection{The basic structure of reaction kinetic networks}

In describing the underlying dynamic structure of reaction networks, we adopt the notation employed in [8] for the dissipative reaction network kinetics. We can distinguish between closed (isolated) and open kinetic networks depending on whether there is or not material exchange with the environment. 
Let us consider the isolated and homogeneous isotherm systems where $n$ chemical species participate on a $r$-step reaction network, represented by the following stoichiometric mechanism:

$$
\sum_{i=1}^{n} \alpha_{i j} A_{i} \rightleftarrows \sum_{i=1}^{n} \beta_{i j} A_{i} \quad \text { for } j=1, \ldots, r
$$

with $\alpha_{i j}, \beta_{i j}$ being the constant stoichiometric coefficients for specie $A_{i}$ in the reaction step $j$. The linear combinations of the species in eq. (1), namely $\sum_{i=1}^{n} \alpha_{i j} A_{i}$ and $\sum_{i=1}^{n} \beta_{i j} A_{i}$ for $j=1, \ldots, r$ are called the complexes. All reactions are assumed to be reversible, with reaction rates obeying the mass action law [7]:

$$
W_{j}(x)=k_{j}^{+} \prod_{i=1}^{n} x_{i}^{\alpha_{i j}}-k_{j}^{-} \prod_{i=1}^{n} x_{i}^{\beta_{i j}}, \quad j=1, \ldots, r
$$

where $k_{j}^{+}$and $k_{j}^{-}$are the constants of the direct and inverse rates of the $j$-th reaction step, respectively, and $x_{i}>0$ represents the concentration of the specie $A_{i}$. Each concentration evolves in time according to the ordinary differential equation:

$$
\dot{x}_{i}=\sum_{j=1}^{r} \nu_{i j} W_{j}(x), \quad i=1, \ldots, n
$$

where $\nu_{i j}=\alpha_{i j}-\beta_{i j}$ is positive or negative depending on whether the specie $i$ is a product or a reactant in the reaction $j$. The dynamic evolution of the network can then be represented by a set of ordinary differential equations which in compact matrix form is written as:

$$
\dot{x}=\mathcal{N} \cdot W(x)
$$

where $\mathcal{N}=\left[\nu_{i j}\right]$ is the $n \times r$ coefficient matrix whose columns are the so-called stoichiometric vectors $\nu_{\cdot j}=\beta_{\cdot j}-\alpha_{\cdot j}$, and $W(x) \in \mathbb{R}^{r}$ denotes the vector of reaction rates.

The following simple example illustrates the above notions. Let the set of species be given by $P_{1}, P_{2}, P_{3}$, and $P_{4}$, while the set of complexes is $P_{1}$, $P_{2}+P_{3}$, and $P_{4}$. The reactions are

$$
P_{1} \underset{k_{1}^{-}}{\stackrel{k_{1}^{+}}{\rightleftarrows}} P_{2}+P_{3} \underset{k_{2}^{-}}{\stackrel{k_{2}^{+}}{\rightleftarrows}} P_{4}
$$


The $\alpha$ and $\beta$ matrices are given by

$$
\alpha=\left[\begin{array}{ll}
1 & 0 \\
0 & 1 \\
0 & 1 \\
0 & 0
\end{array}\right], \beta=\left[\begin{array}{ll}
0 & 0 \\
1 & 0 \\
1 & 0 \\
0 & 1
\end{array}\right]
$$

from which $\mathcal{N}$ can be calculated as

$$
\mathcal{N}=\beta-\alpha=\left[\begin{array}{rr}
-1 & 0 \\
1 & -1 \\
1 & -1 \\
0 & 1
\end{array}\right]
$$

The reaction rates are given by

$$
\begin{aligned}
& W_{1}(x)=k_{1}^{+} x_{1}-k_{1}^{-} x_{2} x_{3} \\
& W_{2}(x)=k_{2}^{+} x_{2} x_{3}-k_{2}^{-} x_{4}
\end{aligned}
$$

Now, let us modify the structure of (5) in such a way that there is a further reaction step between $P_{1}$ and $P_{4}$ :

$$
P_{1} \underset{k_{1}^{-}}{\stackrel{k_{1}^{+}}{\rightleftarrows}} P_{2}+P_{3} \underset{k_{2}^{-}}{\stackrel{k_{2}^{+}}{\rightleftarrows}} P_{4} \underset{k_{3}^{-}}{\stackrel{k_{3}^{+}}{\rightleftarrows}} P_{1}
$$

Now, the modified stoichiometric matrix is

$$
\mathcal{N}^{\prime}=\left[\begin{array}{rrr}
-1 & 0 & 1 \\
1 & -1 & 0 \\
1 & -1 & 0 \\
0 & 1 & -1
\end{array}\right]
$$

and the third reaction rate (in addition to (8) and (9)) reads

$$
W_{3}(x)=k_{3}^{+} x_{4}-k_{3}^{-} x_{1}
$$

Observe that the columns of the original $\mathcal{N}$ are linearly independent which means that $\mathcal{N}$ is of maximal rank. In this case, we say that the elementary reactions (or the stoichiometric vectors) are independent. It is also visible, 
that the rank of the modified $\mathcal{N}^{\prime}$ is only two, because the structure of the modified reaction is such that the third column of $\mathcal{N}^{\prime}$ is a linear combination of the columns of $\mathcal{N}$.

From now, we assume that $r \leq n$ and the the columns of $\mathcal{N}$ are linearly independent i.e., the rank of $\mathcal{N}$ is exactly $r$. With this assumption, we only deal with a proper subclass of closed reversible reaction networks, since many reaction networks occurring in natural or technological systems contain linearly dependent column vectors in $\mathcal{N}$.

The reaction polyhedron. It is easy to see from (4) that any vector $c_{k} \in \mathbb{R}^{n}$, $k=1, \ldots, n-r$ belonging to the kernel of $\mathcal{N}^{T}$ defines a linear first integral:

$$
c_{k}^{T} x(t)=c_{k}^{T} x(0)=: \mathcal{C}_{k}^{0}, \quad \forall t \geq 0, \quad k=1, \ldots, n-r,
$$

since $c_{k}^{T} \dot{x}=0$. It is also known that the positive orthant denoted by $\mathbb{R}_{n}^{+}$is positively invariant for the dynamics (4) (see e.g., [8, 7]). For a given initial condition $x(0)$, equation (13) defines an $r$-dimensional invariant manifold $\mathcal{M}_{x_{0}}$ which is a parallel translate of an $r$-dimensional subspace spanned by the column vectors of $\mathcal{N}$. The intersection of the positive orthant with $\mathcal{M}_{x_{0}}$ is called the reaction polyhedron denoted by $\Omega\left(x_{0}\right)$ :

$$
\Omega\left(x_{0}\right)=\left\{x \in \mathbb{R}_{n}^{+} \mid c_{k}^{T}\left(x-x_{0}\right)=0, \quad k=1, \ldots, n-r\right\},
$$

that is also invariant for the system dynamics.

The equilibrium manifold. From the assumption $r \leq n$ and from the rank condition on $\mathcal{N}$ it follows that $\dot{x}=0 \Leftrightarrow W(x)=0$, which determines the set of equilibrium points of the closed system. It is known from the stability theory of the studied subclass of reaction networks, that within each reaction polyhedron, there is exactly one equlibrium point which is globally stable in the space of positive concentrations (i.e., $\mathbb{R}_{+}^{n}$ ), and globally asymptotically stable if the system's dynamics is restricted to the $r$-dimensional reaction polyhedron. Therefore, assuming that the value of $x(0)$ is known, it is common to study the dynamics of (4) restricted to $\Omega\left(x_{0}\right)$ using the linear equations (13) [15]. From this it also follows that the minimal representation of a chemical reaction system (4) is $r$-dimensional. The equilibrium point of interest will be denoted by $x^{*}$ throughout the paper. 


\subsection{Generalized Hamiltonian systems}

The form of generalized dissipative Hamiltonian systems we will use is defined in [5]. In the autonomous case, this system class is defined by the differential equations

$$
\dot{x}=(J(x)-R(x)) \mathcal{H}_{x}^{T}(x),
$$

where $x \in \mathbb{R}^{n}, \mathcal{H}: \mathbb{R}^{n} \mapsto \mathbb{R}$ is the Hamiltonian function, $J(x)$ is an $n \times n$ skew symmetric matrix (i.e. $J^{T}(x)=-J(x)$ ), the energy conserving part of the system, and $R(x)=R^{T}(x)$ is the so called dissipation matrix. $\mathcal{H}_{x}$ denotes the gradient of $\mathcal{H}$ (row vector).

The time derivative of the Hamiltonian function is

$$
\begin{aligned}
& \dot{\mathcal{H}}=\mathcal{H}_{x}(x)(J(x)-R(x)) \mathcal{H}_{x}^{T}(x)= \\
& \underbrace{\mathcal{H}_{x}(x) J(x) \mathcal{H}_{x}^{T}(x)}_{0}-\mathcal{H}_{x}(x) R(x) \mathcal{H}_{x}^{T}(x) .
\end{aligned}
$$

It is clear from (17) that the skew symmetricity of $J$ implies that $y^{T} J(x) y=$ $0, \forall x, y \in \mathbb{R}^{n}$, which means that $\mathcal{H}$ is a conserved quantity (first integral) of the system if $R=0$. These facts motivate us to use the notion of generalized Hamiltonian systems.

It is also visible from (17) that if $y^{T} R(x) y \geq 0, \forall x, y \in \mathbb{R}^{n}$ (i.e. $R$ is positive semidefinite), then the Hamiltonian function is nonincreasing. Of course, this property might not be satisfied globally, but only in some neighborhood of the equilibrium point.

It is important to note that in physical system models, the matrices $J(x)$ and $R(x)$ often reflect the physical structure or topology of the system [5].

A further important remark clarifies the connection between the generalized Hamiltonian and the GENERIC [16] structures. The general equation for the time-evolution of beyond-equilibrium systems is formalized in the so called GENERIC structure that accounts for the reversible and irreversible contributions of the total energy $E(x)$ and the entropy $S(x)$ :

$$
\dot{x}=L(x) \cdot E_{x}^{T}(x)+M(x) \cdot S_{x}^{T}(x),
$$

where $x$ is a set of independent variables required for a complete description of the system (e.g. energies, velocities, etc.) and $E_{x}$ and $S_{x}$ denote the 
gradients of $E$ and $S$, respectively. In addition, the skew-symmetric matrix $L(x)$ and the positive semi-definite $M(x)$ satisfy the so called degeneracy properties:

$$
\begin{array}{r}
L \cdot S_{x}^{T}(x)=0 \\
M \cdot E_{x}^{T}(x)=0
\end{array}
$$

and the impositions of the First and Second Law of thermodynamics:

$$
\begin{aligned}
& \frac{d E}{d t}=0 \\
& \frac{d S}{d t} \geq 0
\end{aligned}
$$

It is visible that taking into consideration eqs. (19)-(20), the algebraic structure of (15) matches the generic structure (18) with $L=J, M=R$ and $\mathcal{H}$ being a thermodynamic potential constructed from the linear combination of the total energy and entropy of the system. However, the dissipative Hamiltonian structure does not require in general that the Hamiltonian function should be separated into two physically meaningful parts like $E$ and $S$ in the GENERIC framework.

\section{Local Hamiltonian structure of reaction net- works}

Let us denote the forward and backward parts of the reaction rates in the following way

$$
p_{j}(x)=k_{j}^{+} \prod_{i=1}^{n} x_{i}^{\alpha_{i j}}, \quad q_{j}(x)=k_{j}^{-} \prod_{i=1}^{n} x_{i}^{\beta_{i j}}, \quad j=1, \ldots, r
$$

We define the reaction space as follows (the $x$ arguments are suppressed in $p$ and $q)$ :

$$
z_{j}=\ln p_{j}-\ln q_{j}, \quad j=1, \ldots, r
$$

Note that the product $R T z_{j}$ (with $\mathrm{R}$ being the gas constant and $\mathrm{T}$ the temperature) is the chemical affinity corresponding to the reaction step $j[6]$. 
In order to construct an invertible mapping between $x$ and $z$, let us extend (24) with the conserved quantities as additional coordinate functions in the following way

$$
\bar{z}=\Psi(x)
$$

where

$$
\begin{aligned}
& \bar{z}_{j}=z_{j}=\ln \left(\frac{k_{j}^{+}}{k_{j}^{-}} \prod_{i=1}^{n} x_{i}^{\nu_{i j}}\right), \quad j=1, \ldots, r \\
& \bar{z}_{k}=c_{k-r}^{T} \cdot x, \quad k=r+1, \ldots, n
\end{aligned}
$$

We assume that we know the value of $x$ at any time instant, therefore $\bar{z}_{k}$ in (27) are known and constant. Furthermore, it is assumed that the Jacobian of $\Psi$ is full rank at any $x_{i}>0, i=1, \ldots, n$ and this implies that the inverse $x=\Psi^{-1}(z)$ exists in the whole positive orthant.

The time-derivative of $z_{j}$ is given by

$$
\begin{aligned}
& \dot{z}_{j}=\frac{1}{p_{j}} \dot{p}_{j}-\frac{1}{q_{j}} \dot{q}_{j}=\frac{1}{p_{j}} \frac{\partial p_{j}}{\partial x} \dot{x}-\frac{1}{q_{j}} \frac{\partial q_{j}}{\partial x} \dot{x}= \\
& =\left[\begin{array}{llll}
\frac{1}{x_{1}}\left(\alpha_{1 j}-\beta_{1 j}\right) & \frac{1}{x_{2}}\left(\alpha_{2 j}-\beta_{2 j}\right) & \ldots & \frac{1}{x_{n}}\left(\alpha_{n j}-\beta_{n j}\right)
\end{array}\right] \dot{x}
\end{aligned}
$$

Using (4) and (28), the time derivative of the vector $z$ can be written as

$$
\dot{z}=-\mathcal{N}^{T} \Gamma(x) \cdot \dot{x}=-\mathcal{N}^{T} \Gamma(x) \mathcal{N} \cdot W(x),
$$

where

$$
\Gamma(x)=\operatorname{diag}\left(\frac{1}{x_{1}}, \frac{1}{x_{2}}, \ldots, \frac{1}{x_{n}}\right) .
$$

The rank of $\mathcal{N}$ is always $r$ and $x_{i}>0$ for $i=1, \ldots, n$, therefore the matrix $\mathcal{N}^{T} \Gamma(x) \mathcal{N}$ is nonsingular and positive definite at any fixed $x$, since it can be written as $P^{T} P$, where

$$
P=(\Gamma(x))^{1 / 2} \mathcal{N}
$$

This means, that the system (29) is at equilibrium if and only if $W(x)=0$ i.e. the unique equilibrium point in the $z$-coordinates is at $z_{i}=0, i=1, \ldots, r$.

Furthermore, from eqs. (2), (23) and (24) the reaction rates can be expressed as

$$
W_{j}=p_{j}-q_{j}=\exp \left(z_{j}\right) q_{j}-q_{j}=q_{j}\left[\exp \left(z_{j}\right)-1\right]
$$


Let us use the following notation:

$$
F(x)=\operatorname{diag}\left(q_{1}(x), \ldots, q_{r}(x)\right)
$$

Let the Hamiltonian function $H$ be defined as

$$
H(z)=\sum_{j=1}^{r} q_{j}^{*}\left[\exp \left(z_{j}\right)-z_{j}-1\right]
$$

where $q_{i}^{*}$ denotes the value of $q_{i}$ at the equilibrium point $x^{*}$. It is easy to show that $H$ is globally convex and bounded from below, therefore it can be used as a Lyapunov function.

Using Eqs. (29), (32) and (34) we can write

$$
\dot{z}=-\mathcal{N}^{T} \Gamma(x) \mathcal{N} \cdot F(q) \cdot\left(F\left(q^{*}\right)\right)^{-1} \cdot H_{z}^{T}(z)
$$

or shortly

$$
\dot{z}=-G(x) \cdot H_{z}^{T}(z)
$$

where

$$
G(x)=\mathcal{N}^{T} \Gamma(x) \mathcal{N} \cdot F(q) \cdot\left(F\left(q^{*}\right)\right)^{-1}
$$

and $H_{z}^{T}$ denotes the gradient transpose of $H$. Using the assumption that $\Psi$ is invertible, (36) can be written as

$$
\dot{z}=-G\left(\Psi^{-1}(\bar{z})\right) \cdot H_{z}^{T}(z)
$$

It is easy to see, that $G+G^{T}$ is positive definite in a neighborhood of the equilibrium point, since it is smooth with respect to $x$ in the positive orthant and

$$
G\left(x^{*}\right)=\mathcal{N}^{T} \Gamma\left(x^{*}\right) \mathcal{N} \cdot F\left(q^{*}\right) \cdot\left(F\left(q^{*}\right)\right)^{-1}=\mathcal{N}^{T} \Gamma\left(x^{*}\right) \mathcal{N}
$$

However, it cannot be guaranteed for an arbitrary reaction network that $G+G^{T}$ is positive definite in the whole concentration space. Therefore we can state the following proposition

Proposition 1 For any closed reaction kinetic system of the form (4) with independent elementary reactions, there exists a neighborhood $U$ around the equilibrium point $z^{*}=0$, where the system admits a dissipative Hamiltonian description with Hamiltonian function (34). 
Physical interpretation The $\mathrm{G}$ matrix in the Hamiltonian description reflects the connectivity properties of the reaction network $(\mathcal{N}$ together with the chemical composition of the complexes defines the graph structure of the system). It is also visible that $G\left(x^{*}\right)$ is symmetric and $G$ looses its symmetry outside the equilibrium point. The Hamiltonian function $\mathcal{H}$ contains the scaled chemical affinities, but it is rather an abstract construction and not the total energy of the system as in the case of most mechanical and electrical systems.

In a thermodynamic sense, the local definiteness of $G+G^{T}$ is related to the so-called entropy production function given by the product of thermodynamic fluxes and forces:

$$
\dot{B}=-z^{T} \cdot W=-\sum_{j=1}^{r} \ln \frac{p_{j}}{q_{j}}\left(p_{j}-q_{j}\right)
$$

where the transformed entropy function $B$ in the space of the chemical concentrations is defined as

$$
B(x)=\sum_{i=1}^{n} x_{i}\left(\ln \left(\frac{x_{i}}{x_{i}^{*}}\right)-1\right)+x_{i}^{*}
$$

It is known that $B$ (which is a measure of the inner dissipation of the system) is globally convex in the positive orthant and it is nonincreasing in time (see, e.g. [8]). Therefore $\dot{B}$ is globally negative semidefinite but it is only locally concave (as a function of the concentrations) in a neighborhood of the equilibrium point determined by the topology of the reaction network and the kinetic constants. $\dot{B}$ loses its concavity in the so called far from equilibrium region of the concentration space [12]. This property can be a source of complex nonlinear behavior (e.g. multiple steady states or nonlinear oscillations) when the system is opened and the exchange of material with its environment is permitted that can be modeled as external manipulable (or disturbance) inputs [14]. 


\section{Examples}

\subsection{Global dissipative Hamiltonian description}

Let the reaction system with six species $P_{11}, P_{12}, P_{13}, P_{21}, P_{22}$, and $P_{23}$ (together with the corresponding concentrations $x_{1}, x_{2}, \ldots, x_{6}$, respectively) and four complexes $P_{11}+P_{12}, P_{13}, P_{21}+P_{22}$ and $P_{23}$ be given in the following form.

$$
\begin{aligned}
& P_{11}+P_{12} \underset{k_{1}^{-}}{\stackrel{k_{1}^{+}}{\rightleftarrows}} P_{13} \\
& P_{21}+P_{22} \underset{k_{2}^{-}}{\stackrel{k_{2}^{+}}{\rightleftarrows}} P_{23}
\end{aligned}
$$

The matrices characterizing the reaction network are given by

$$
\begin{gathered}
\mathcal{N}^{T}=\left[\begin{array}{llllll}
-1 & -1 & 1 & 0 & 0 & 0 \\
0 & 0 & 0 & -1 & -1 & 1
\end{array}\right] \\
\Gamma(x)=\operatorname{diag}\left(\frac{1}{x_{1}}, \ldots, \frac{1}{x_{6}}\right), \quad F(x)=\operatorname{diag}\left(q_{1}(x), q_{2}(x)\right),
\end{gathered}
$$

and

$$
G(x)=\left[\begin{array}{cc}
\frac{\left(x_{1}^{-1}+x_{2}^{-1}+x_{3}^{-1}\right) x_{3}}{x_{3}^{*}} & 0 \\
0 & \frac{\left(x_{4}^{-1}+x_{5}^{-1}+x_{6}^{-1}\right) x_{6}}{x_{6}^{*}}
\end{array}\right]
$$

We can see that $G$ is globally positive definite in the whole positive orthant, which means that the reaction system admits a global dissipative Hamiltonian structure. Based on the above calculations, it is easy to see that any set of independent reactions of the type

$$
\sum_{j=1}^{\delta_{i}} c_{i j} P_{i j} \underset{k_{i}^{-}}{\stackrel{k_{i}^{+}}{\rightleftarrows}} \sum_{l=\delta_{i}+1}^{n_{i}} c_{i l} P_{i l}, \quad \delta_{i}<n_{i}, \quad i=1, \ldots, r
$$

defines a global dissipative Hamiltonian structure. 


\subsection{Local dissipative Hamiltonian description}

\subsubsection{Model of the reaction network}

Consider the following simple reaction network with three species $\left(P_{1}, P_{2}\right.$, $\left.P_{3}\right)$ and four complexes $P_{1}+P_{2}, P_{3}, P_{2}+P_{3}$ and $2 P_{3}$ :

$$
\begin{aligned}
& P_{1}+P_{2} \underset{k_{1}^{-}}{\stackrel{k_{1}^{+}}{\rightleftarrows}} P_{3} \\
& P_{2}+P_{3} \underset{k_{2}^{-}}{\stackrel{k_{2}^{+}}{\rightleftarrows}} 2 P_{3}
\end{aligned}
$$

The matrix $\mathcal{N}$ of the system is written as

$$
\mathcal{N}=\left[\begin{array}{rr}
-1 & 0 \\
-1 & -1 \\
1 & 1
\end{array}\right]
$$

The forward and backward reaction rates are

$$
\begin{aligned}
& p_{1}=k_{1}^{+} x_{1} x_{2}, q_{1}=k_{1}^{-} x_{3} \\
& p_{2}=k_{2}^{+} x_{2} x_{3}, q_{2}=k_{2}^{-} x_{3}^{2}
\end{aligned}
$$

From the above equations, the components of the vector $W$ are calculated as

$$
\begin{aligned}
& W_{1}=p_{1}-q_{1}=k_{1}^{+} x_{1} x_{2}-k_{1}^{-} x_{3} \\
& W_{2}=p_{2}-q_{2}=k_{2}^{+} x_{2} x_{3}-k_{2}^{-} x_{3}^{2}
\end{aligned}
$$

Using $N$ and $W$, the equations of the reaction system are the following

$$
\begin{aligned}
& \dot{x}_{1}=k_{1}^{-} x_{3}-k_{1}^{+} x_{1} x_{2} \\
& \dot{x}_{2}=k_{1}^{-} x_{3}-k_{1}^{+} x_{1} x_{2}+k_{2}^{-} x_{3}^{2}-k_{2}^{+} x_{2} x_{3} \\
& \dot{x}_{3}=k_{1}^{+} x_{1} x_{2}-k_{1}^{-} x_{3}+k_{2}^{+} x_{2} x_{3}-k_{2}^{-} x_{3}^{2}
\end{aligned}
$$




\subsubsection{Dissipative Hamiltonian structure of the closed system}

The coordinates-transformation $\Psi$ is given by

$$
\begin{aligned}
& \bar{z}_{1}=\ln \left(\frac{p_{1}}{q_{1}}\right)=\ln \left(\frac{k_{1}^{+}}{k_{1}^{-}} x_{1} x_{2} x_{3}^{-1}\right) \\
& \bar{z}_{2}=\ln \left(\frac{p_{2}}{q_{2}}\right)=\ln \left(\frac{k_{2}^{+}}{k_{2}^{-}} x_{2} x_{3}^{-1}\right) \\
& \bar{z}_{3}=x_{2}+x_{3}
\end{aligned}
$$

Then the inverse transformation $\Psi^{-1}$ can be calculated as

$$
\begin{aligned}
x_{1} & =\frac{k_{2}^{+} k_{1}^{-}}{k_{2}^{-} k_{1}^{+}} \exp \left(\bar{z}_{1}-\bar{z}_{2}\right) \\
x_{2} & =\frac{k_{2}^{-} z_{3} \exp \left(\bar{z}_{2}\right)}{k_{2}^{-} \exp \left(\bar{z}_{2}\right)+k_{2}^{+}} \\
x_{3} & =\frac{k_{2}^{+} \bar{z}_{3}}{k_{2}^{-} \exp \left(\bar{z}_{2}\right)+k_{2}^{+}}
\end{aligned}
$$

We can see from eqs. (58)-(63) that $\Psi$ and $\Psi^{-1}$ are globally defined in the positive orthant of the space of concentrations.

The dissipative Hamiltonian structure (36) for the model (55)-(57) is computed as

$$
\left[\begin{array}{l}
\dot{z}_{1} \\
\dot{z}_{2}
\end{array}\right]=-\left[\begin{array}{ll}
\frac{1}{x_{1}}+\frac{1}{x_{2}}+\frac{1}{x_{3}} & \frac{1}{x_{2}}+\frac{1}{x_{3}} \\
\frac{1}{x_{2}}+\frac{1}{x_{3}} & \frac{1}{x_{2}}+\frac{1}{x_{3}}
\end{array}\right] \cdot\left[\begin{array}{cc}
\frac{x_{3}}{x_{3}^{*}} & 0 \\
0 & \frac{x_{3}^{2}}{\left(x_{3}^{*}\right)^{2}}
\end{array}\right] \cdot H_{z}^{T}(z)
$$

where

$$
H_{z}=\left[\begin{array}{ll}
k_{1}^{-} x_{3}^{*}\left(\exp \left(z_{1}\right)-1\right) & k_{2}^{-}\left(x_{3}^{*}\right)^{2}\left(\exp \left(z_{2}\right)-1\right)
\end{array}\right]
$$

For the forthcoming calculations, the values of the reaction rate constants $k_{1}^{+}, k_{1}^{-}, k_{2}^{+}, k_{2}^{-}$were chosen to be uniformly 1 . The dissipativity region (i.e. the region inside which the matrix $G+G^{T}$ is positive definite) and the level sets of $H$ for $x^{*}=\left[\begin{array}{lll}1 & 5 & 5\end{array}\right]^{T}$ in the $\left(x_{1}, x_{2}\right)$ and $\left(z_{1}, z_{2}\right)$ planes can be seen in Figs 1 and 2, respectively. It is visible that the dissipative Hamiltonian description is valid in a wide neighborhood of the equilibrium point for the selected reaction polyhedron. 


\section{Conclusions}

It has been shown in this paper that closed reversible reaction networks with independent elementary reactions admit a global pseudo Hamiltonian and a local dissipative Hamiltonian structure with clear physical meaning around any equilibrium point. The Hamiltonian structure has been described in a transformed coordinates system called the reaction space which is generally of lower dimension than the concentration space. The structure matrix which is a smooth function of the concentrations depends on the topology of the reaction network and the coefficients of the Hamiltonian function depend on the reaction polyhedron on which the system dynamics evolve. The theoretical results have been illustrated by two simple examples.

\section{Acknowledgements}

The authors acknowledge financial support received from the Spanish Government (MCyT Projects PPQ2001-3643 \& DPI2004-07444-C04-03) and Xunta de Galicia (PGIDIT02-PXIC40209PN). This research was partially supported by the Hungarian grants no. K67625, F046223, which are gratefully acknowledged. The second author is a grantee of the Bolyai János Research Scholarship of the Hungarian Academy of Sciences.

\section{References}

[1] A.A. Alonso and B.E. Ydstie. Stabilization of distributed systems using irreversible thermodynamics. Automatica, 37:1739-1755, 2001.

[2] D. Cheng, A. Astolfi, and R. Ortega. On feedback equivalence to port controlled Hamiltonian systems. Systems and Control Letters, 54:911$917,2005$.

[3] D. Cheng, T. Shen, and T.J. Tarn. Pseudo-Hamiltonian realization and its application. Communications in Information and Systems, 2:91-120, 2002 . 
[4] J. Clemente-Gallardo and J.M.A. Scherpen. Relating Lagrangian and Hamiltonian framework for LC circuits. IEEE Trans. Circ. and Syst., 50:1359-1363, 2003.

[5] A.J. Van der Schaft. L2-Gain and Passivity Techniques in Nonlinear Control. Springer Verlag, Berlin, 2000.

[6] T. De Donder. Thermodynamic Theory of Affinity: A Book of Principles. Oxford University Press, 1936.

[7] M. Feinberg. Lectures on chemical reaction networks. Notes of lectures given at the Mathematics Research Center, University of Wisconsin, 1979 .

[8] A.N. Gorban, I.V. Karlin, and A.Y. Zinovyev. Invariant grids for reaction kinetics. Physica A, 33:106-154, 2004.

[9] R. Lopezlena and J.M.A. Scherpen. On distributed port-Hamiltonian process systems. In Proc. 6th IFAC Symposium on Nonlinear Control Systems (NOLCOS 2004), pages 1253-1258, 2004.

[10] B.M. Maschke, A.J. Van der Schaft, and P.C. Breedveld. An intrinsic Hamiltonian formulation of the dynamics of LC-circuits. IEEE Trans. Circ. and Syst., CAS-42:73-82, 1995.

[11] R.I. McLachlan, G.R.W. Quispel, and N. Robidoux. Unified approach to Hamiltonian systems, Poisson systems, gradient systems and systems with Lyapunov functions or first integrals. Physical Review Letters, 81:2399-2403, 1998.

[12] G. Nicolis and I. Prigogine. Self-organization in Nonequilibrium Systems: From Dissipative Structures to Order through Fluctuations. John Wiley \& sons, New York, 1977.

[13] R. Ortega, A.J. van der Schaft, and B.M. Maschke. Stabilization of portcontrolled Hamiltonian systems via energy balancing. Lecture Notes in Control and Information Sciences, 246:239-260, 1999. 
[14] I. Otero-Muras, G. Szederkényi, K.M. Hangos, and A.A. Alonso. Dynamic analysis and control of biochemical reaction networks. Mathematics and Computers in Simulation, 2007. to appear.

[15] E. Sontag. Structure and stability of certain chemical networks and applications to the kinetic proofreading model of T-cell receptor signal transduction. IEEE Trans. Autom. Control, 46:1028-1047, 2001.

[16] H.C. Öttinger. Beyond Equilibrium Thermodynamics. John Wiley \& Sons, 2005.

[17] Y. Wang, D. Cheng, and S.S. Ge. Approximate dissipative Hamiltonian realization and construction of local Lyapunov functions. Systems and Control Letters, 2006. doi: 10.1016/j.sysconle.2006.08.005.

[18] B.E. Ydstie and A.A. Alonso. Process systems and passivity via the Clausius-Planck inequality. Systems and Control Letters, 30:253-264, 1997. 


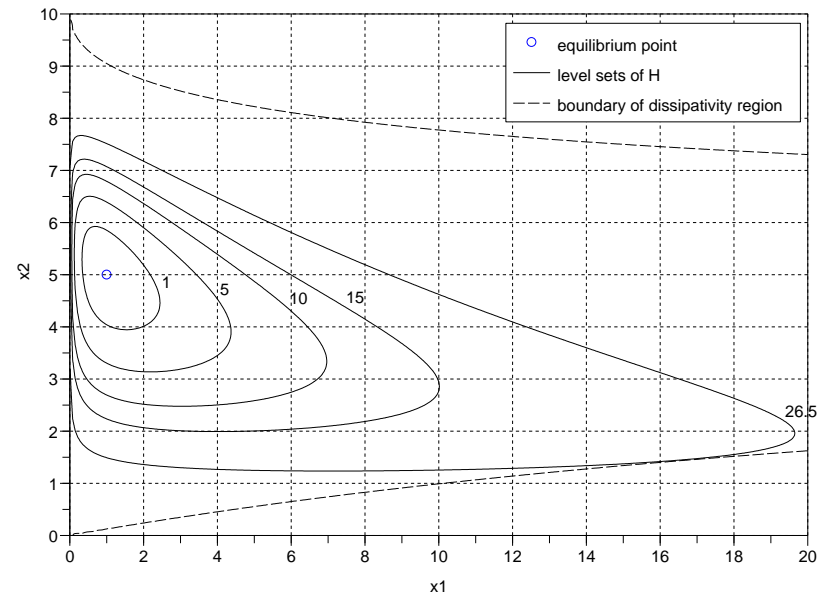

Figure 1: Dissipativity region and level sets of the Hamiltonian function in the $\left(x_{1}, x_{2}\right)$ plane

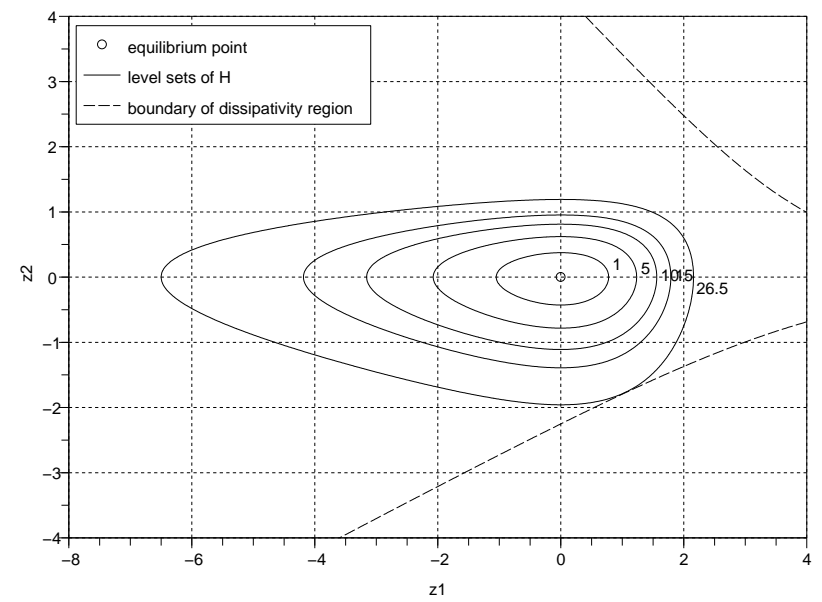

Figure 2: Dissipativity region and level sets of the Hamiltonian function in the $\left(z_{1}, z_{2}\right)$ plane 